\title{
Fifteen minutes
}

\author{
QUINZE MINUTOS
}

Wanderley Bernardo, José Maria Jr., Antônio Salomão, Edmund Baracat

http://dx.doi.org/10.1590/1806-9282.60.05.001

\section{DESCRIBING THE IMPORTANCE OF OBESITY TODAY, AND THE LACK OF STRATEGIES FOR PROPER MA- NAGEMENT, CAN TAKE FIFTEEN MINUTES OF REA- DING, BUT THE PROBLEM MUST INVOLVE MUCH MORE TIME OF YOUR ATTENTION, AS WELL AS GUI- DANCE TO YOUR PATIENTS.}

Epidemic and rising rates of obesity in many parts of the world are leading to increased suffering and economic stress. Despite decades of research on the causes of the obesity pandemic, which does not seem close to a solution, there is still no clear understanding of the nature of the problem. This limits creativity and suffocates expansive thinking, which could advance in the field of prevention and treatment, as well as in the scope of the complications of obesity. Shared decision-making and the redirecting of policies could remove barriers that prevent us from moving forward to solve an urgent public health issue of the beginning of this century ${ }^{1.2}$.

Overweight and obesity reflect a gain of excess body fat, including visceral fat, which is a result of initially imperceptible cumulative effects of everyday eating, hourly, with no proper physical activity, creating a surplus of calories consumed in relation to those expended. Excess weight gain, gradual and unintended, is accompanied by the difficulty of reversing the picture and can become permanent. Even if all currently obese patients were treated effectively in the absence of adequate preventive efforts, there would still be continued growth in the number of obese people. Many multifaceted interventions to prevent obesity seek to influence the balance of calories, focusing on energy consumption, or energy expenditure. While obesity is a priority from an epidemiological and public health perspective, it becomes even more important as it influences other aspects of society. Substantial direct and indirect costs include discrimination, economic deprivation, loss of productivity and disability. Thus, state and local governments end up diverting resources for prevention and treatment. The country's health system is burdened with the comorbidities of obesity, such as type 2 diabetes, hypertension, cardiovascular diseases, osteoarthritis and cancer. It is estimated that the annual burden of obesity is almost $10 \%$ of all medical spending 3 .

There are programs of shared decision-making that offer surgical treatment modalities for weight loss, including:
Roux-en-Y gastric bypass, laparoscopic adjustable gastric banding and laparoscopic sleeve gastrectomy. Patients attend seminars where they are instructed on the differences in outcomes, follow-up and complications of each procedure. The main information presented at the seminar include: 1. Greater weight loss with Roux-en-Y and sleeve gastrectomy compared to the use of a gastric band; 2 . The gastric band requires the highest number of post-operative visits (monthly in the first year); 3 . The Roux-en-Y and the sleeve gastrectomy have a higher rate of life-threatening complications than the gastric band (fistula); 4. The gastric band has highest number of delayed complications related to the device (erosion, migration); 5. The Roux-en-Y has the highest rate of diabetes remission; and 6 . There is a lack of data on five-year follow-up of weight loss after sleeve gastrectomy. Fifty-eight percent of patients chose "weight loss" as the most important result, and $65 \%$ chose "fistula" as the most worrisome complication. A subgroup analysis including patients with diabetes showed that $58 \%$ chose "curing diabetes" as the most important result. Nineteen percent of patients were unsure about which procedure they wanted, or changed their decision after consultation with the surgeon ${ }^{3}$.

\section{THE CHOICE FOR BARIATRIC SURGERY IS NOT EASY, AND SHARED DECISION-MAKING IN THE MANAGE- MENT OF OBESITY SHOULD ADDRESS BOTH THE ME- DICAL AND SURGICAL TREATMENT, AND PREVEN- TION, REQUIRING MORE THAN FIFTEEN MINUTES.}

In decision-making, patients can choose to be more passive. That is because they do not know how to feel when they are more active in decisions. Or, they may fear abandonment or being labeled "difficult if they seem to defy the doctor's authority. They may find it difficult to tolerate the doubt, being satisfied with the first solution, less than ideal, but available. In turn, physicians may assume that the patient made the decision based on correct information and appropriate assumptions, especially if the professional opinion is naturally favored. It is generally accepted that whenever patients are well-informed, they can make the best decisions. Patients often want more information than expected. Sometimes, however, the more information is presented, the worse is the understanding of patients. Patients and physicians need to consider the right amount. In addition, the number and types of suitable options (not all possible options at once) and 
the right time to accommodate the limits of individual and shared cognitive processing. Physicians should be alert to the potential of hierarchical relationships that can promote coercion or silence the voice of the patient, and regularly check for understanding. They should also be aware of the indirect signs of emotional distress and demonstrate reliability and transparency, actively soliciting the patient's questions and concerns. Self-knowledge, self-monitoring, honesty and the willingness to challenge one's own assumptions are key qualities, which, when cultivated by the physician, will help him/her distinguish his/her participation in increasing autonomy at the expense of well-meaning, but mistaken, imposition of values 4 .

\section{Proper patient care requires dialogue With REGARD TO OPTIONS AND DECISIONS, WHICH IS NOT ACCOMPLISHED IN 15 MINUTES.}

- Talking about choices. How to get patients to understand that there are reasonable options? The components of this stage are: a) Step one - Summarize and say: "Now that we have identified the problem, it's time to think about what to do next"; b) Offer choices - Please note that patients often misinterpret the presentation of choices and think the doctor is either incompetent or uninformed, or both. Reduce this risk by saying: "There is good information about how these treatment options differ, and I would like to discuss this with you"; c) Justify the choice - Emphasize: 1) The importance of respecting individual preferences; 2) The role of uncertainty. Customize preferences: explain that different issues are more important to some people than to others, which should be easily understood. Say: "Treatments have different consequences. Some will be more important to you than to others." Uncertainty: Patients often are unaware of the extent of uncertainty in medicine, how evidence can be weak and the results, unpredictable at the individual level. Say: "Treatments are not always effective, and the chances of suffering side effects vary." d) Check the patient's reaction - The choice among options can be confusing: some patients may express concern. Suggested phrases: "Let's continue" or "Should I tell you about the options"? e) Postpone the end of the conversation - Some patients react asking doctors: "Tell me what to do." Postpone the end in case this occurs, assuring your patient that you are willing to support his/her decision-making process. Say: "I'm happy to share my opinions and help you reach a good decision. But before I do that, can I describe the options in more detail so that you understand what is at stake?5

- Talking about the options: a) Check their knowledge - Even well-informed patients may be only partially awa- re of the options, and the harms and benefits associated with the procedures, or they may be misinformed. Evaluate with the question: "What have you heard or read about the treatment of obesity?"; b) Write the options Make a clear list of alternatives, as this offers good structure. Say: "Let me list the options before we go into more detail". If this is the case, add the option "wait watching" or use positive terms such as "active monitoring"; c) Describe the options (in practical terms) - Generate dialogue and explore preferences. If there are two medical treatments, says: "Both options are similar and involve taking medication regularly." Point out when there are clear differences (surgery or medication), situations in which it is possible to postpone, or those in which decisions are reversible. Say: "These options have different implications for you in relation to others, and so I want to describe ...". Harm and benefits - Being clear about the pros and cons of the different options is crucial in shared decision-making. Learn effective communication about risk, effects of the process, importance of providing data on absolute risk, as well as in relative terms; d) Support the patient's decision - Synthetic tools make the options visible and can save time. Some are sufficiently concise to use in clinical visits. Examples: cards related to the subject, decision charts and option tables. Shared decision may need more than a medical visit. More extensive tools for patient decision support can play a crucial role. Say: "These tools are designed to help you understand the options in more detail. Use them and come back so that I can answer your questions." e) Summarize - List the options again and assess understanding, asking reformulations ${ }^{5}$.

- Talking about the decision: a) Focus on preferences Guide the patient to express them. Suggested phrases: "What, from your point of view, is more important to you?" b) Inducing a preference - Be prepared to provide additional time or willing to guide the patient if he/she indicates that this is his/her wish; c) Move to a decision - Try to verify the need to postpone the decision or take it. Suggested phrases: "Are you ready to decide?" / "Do you want more time?" / "Do you have any other questions?" / "Is there anything else that we need to discuss?" d) Review the offer - Reminding the patient, whenever possible, that decisions can be reviewed is a good way to end the conversation ${ }^{5}$.

Briefly, patients must decide not only through a theoretical exercise, but considering the context that preserves the expression of their autonomy, in which there must be: clarity on where the care is provided; about the treatment process or outcome to achieve a particular health 
status; adequate information regarding their preferences; perceived credibility of the information source; clarity in the language used to describe options; attention to different attributes of a decision such as benefits and harms; number of options available; valuing of previous experiences in similar situations; affection; description of the effects in the order in which they occur; analysis of reliable opinions of others; perceived social norms; and media influence ${ }^{6}$.

Modalities that lead to rapid, invasive and irreversible solutions used in the treatment of obesity, which grow exceeding their applications, occupy the space of absence or failure of preventive measures and medical treatment.

Reading this walkthrough of shared decision-making can be done in just fifteen minutes. However, in order to care for patients who suffer, and health systems that do not know how to preserve them, medicine is in urgent need of much more than fifteen minutes of dedication. The consequences of a poor decision can last more than fifteen minutes, hours, days, months, years or decades.

\section{References}

1. Hebert JR, Allison DB, Archer E, Lavie CJ, Blair SN. Scientific decision making, policy decisions, and the obesity pandemic. Mayo Clin Proc 2013; 88: 593-604.

2. Institute of Medicine (US) Committee on an Evidence Framework for Obesity Prevention Decision Making; Kumanyika SK, Parker L, Sim LJ, editors. Bridging theEvidence Gap in Obesity Prevention: A Framework to Inform Decision Making.Washington (DC): National Academies Press (US); 2010.

3. Weinstein AL, Marascalchi BJ, Spiegel MA, Saunders JK, Fagerlin A, Parikh M. Patient Preferences and Bariatric Surgery Procedure Selection; the Need for Shared Decision-Making. Obes Surg 2014.

4. Epstein RM. Whole mind and shared mind in clinical decision-making. Patient Educ Couns 2013; 90: 200-6.

5. Elwyn G, Frosch D, Thomson R, Joseph-Williams N, Lloyd A, Kinnersley P, et al. Shared decision making: a model for clinical practice. J Gen Intern Med 2012; 27:1361-7.

6. Epstein RM, Gramling RE. What is shared in shared decision making? Complex decisions when the evidence is unclear. Med Care Res Rev 2013; 70 (1 Suppl): 94S-112S. 